\title{
Asymptotic expansions for Riesz potentials of Airy functions and their products
}

\author{
Nico M Temme ${ }^{1}$ and Vladimir Varlamov ${ }^{2}$ \\ ${ }^{1}$ CWI, P.O. Box 94079, 1090 GB Amsterdam, The Netherlands \\ ${ }^{2}$ Department of Mathematics, University of Texas - Pan American, Edinburg, TX \\ 78539-2999, USA \\ E-mail: Nico.Temme@cwi.nl, Varlamov@utpa.edu
}

\begin{abstract}
Riesz potentials of a function are defined as fractional powers of the Laplacian. Asymptotic expansions for $x \rightarrow \pm \infty$ are derived for the Riesz potentials of the Airy function $A i(x)$ and the Scorer function $G i(x)$. Reduction formulas are provided that allow to compute Riesz potentials of the products of Airy functions $A i^{2}(x)$ and $A i(x) B i(x)$, where $B i(x)$ is the Airy function of the second type, via the Riesz potentials of $A i(x)$ and $G i(x)$. Integral representations are given for the function $A_{2}(a, b ; x)=A i(x-a) A i(x-b)$ with $a, b \in \mathbf{R}$, and its Hilbert transform. Combined with the above asymptotic expansions they can be used for obtaining asymptotics of the Hankel transform of Riesz potentials of $A_{2}(a, b ; x)$. The study of the above Riesz fractional derivatives can be used for establishing new properties of Korteweg-de Vries-type equations.
\end{abstract}

PACS numbers: 02.30.Gp, 02.30.Jr, 02.30.Lt, 02.30.Uu

AMS classification scheme numbers: 35K55, 35L75, 33C10, 41A60

\section{Introduction}

It is well known that fundamental solutions of equations of the Korteweg-de Vries (KdV henceforth) type are expressed in terms of the Airy function of the first type $A i(x)$. Indeed, the fundamental solution of the linearized Cauchy problem for the classical Korteweg-de Vries equation,

$$
u_{t}+u_{x x x}=-\left(u^{2}\right)_{x},
$$

can be written in the form

$$
\mathcal{E}_{0}(x, t)=\frac{1}{\sqrt[3]{3 t}} A i\left(\frac{x}{\sqrt[3]{3 t}}\right) .
$$

It was shown in [1] that for the close relative of $\mathrm{KdV}$, the Ostrovsky equation,

$$
u_{t}+u_{x x x}=\gamma \int_{-\infty}^{x} u \mathrm{~d} y-\left(u^{2}\right)_{x}
$$


where $\gamma=$ const $>0$ is the rotation parameter, the corresponding fundamental solution can be represented in the form

$$
\begin{aligned}
\mathcal{E}(x, t)=-\frac{1}{\sqrt[3]{3 t}} & \frac{\mathrm{d}}{\mathrm{d} x} \int_{0}^{\infty} A i\left(\frac{x+y}{\sqrt[3]{3 t}}\right) J_{0}(2 \sqrt{\gamma t y}) \mathrm{d} y \\
& =\frac{1}{\sqrt[3]{3 t}} A i\left(\frac{x}{\sqrt[3]{3 t}}\right)-\frac{\sqrt{\gamma t}}{\sqrt[3]{3 t}} \int_{0}^{\infty} A i\left(\frac{x+y}{\sqrt[3]{3 t}}\right) \frac{J_{1}(2 \sqrt{\gamma t y})}{\sqrt{y}} \mathrm{~d} y
\end{aligned}
$$

where $J_{\nu}(x)$ is the Bessel function of order $\nu$.

Riesz potentials (sometimes also called Riesz fractional derivatives) of fundamental solutions are of great importance in studying global solvability, properties and the long-time behavior of the corresponding Cauchy problems (see $[2,3,4,5]$ and the references therein). In the current paper we are concerned with obtaining asymptotic expansions as $x \rightarrow \pm \infty$ of the Riesz potentials of the Airy function $A i(x)$ and the Scorer function $G i(x)=-H A i(x)$, where $H$ is the Hilbert transform (see (5) below). Riesz fractional derivatives of these functions of order $\alpha=1 / 2$ stand out as the highest Riesz potentials that are still uniformly bounded on the whole real axis (see $[2,3]$ ). Moreover, all semi-integer derivatives of $A i(x)$ and $G i(x)$ can be expressed in terms of the products of Airy functions (see [5]). We also provide formulas that allow one to obtain asymptotic expansions of the products of Airy functions $A i(x) B i(x), A i^{2}(x)$ and $A i(x-a) A i(x-b)$ with $a, b \in R$. Here $B i(x)$ is the Airy function of the second type.

The next statement was proved in [6]. It provides reduction formulas that allow to compute Riesz potentials of the products of Airy functions once $D_{x}^{\alpha} A i(x)$ and $D_{x}^{\alpha} G i(x)$ are known.

Theorem 1 Riesz fractional derivatives of the products of Airy functions have the following representations for $\alpha>-1 / 2$ and $x \in \mathbf{R}$ :

$$
\begin{aligned}
D_{x}^{\alpha}\left\{A i^{2}(x)\right\}= & k_{\alpha}\left[\left(D^{\alpha-1 / 2} A i\right)\left(2^{2 / 3} x\right)\right. \\
& \left.-\left(D^{\alpha-1 / 2} G i\right)\left(2^{2 / 3} x\right)\right]
\end{aligned}
$$

and

$$
\begin{gathered}
D_{x}^{\alpha}\{A i(x) B i(x)\}=k_{\alpha}\left[\left(D^{\alpha-1 / 2} A i\right)\left(2^{2 / 3} x\right)\right. \\
\left.+\left(D^{\alpha-1 / 2} G i\right)\left(2^{2 / 3} x\right)\right]
\end{gathered}
$$

where

$$
k_{\alpha}=\frac{2^{2(\alpha-1) / 3}}{\sqrt{2 \pi}} .
$$

\section{Definitions}

The Fourier transform of the function $f: \mathbf{R} \rightarrow \mathbf{R}$ is defined by the formula

$$
\hat{f}(\xi)=\mathcal{F}\{f\}(\xi)=\int_{-\infty}^{\infty} \mathrm{e}^{-\mathrm{i} \xi x} f(x) \mathrm{d} x
$$


and the inverse Fourier transform by

$$
f(x)=\mathcal{F}^{-1}\{\hat{f}\}(x)=\frac{1}{2 \pi} \int_{-\infty}^{\infty} \mathrm{e}^{\mathrm{i} \xi x} \hat{f}(\xi) \mathrm{d} \xi .
$$

Introduce the Hankel transform of the function $f$ by the formula (see [7, p. 316])

$$
\tilde{f}(k)=\mathcal{H}_{x \rightarrow k}\{f\}(k)=\int_{0}^{\infty} f(x) J_{m}(k x) x \mathrm{~d} x
$$

and the corresponding inverse transform by

$$
\mathcal{H}_{k \rightarrow x}^{-1}\{\tilde{f}\}(x)=\int_{0}^{\infty} \tilde{f}(k) J_{m}(k x) k \mathrm{~d} k .
$$

Introduce the Hilbert transform of the function $f$ by the formula (see [8, p. 120])

$$
H\{f\}(x)=\frac{1}{\pi} P . V . \int_{-\infty}^{\infty} \frac{f(y)}{y-x} \mathrm{~d} y,
$$

where $x \in \mathbf{R}$ and P.V. denotes the Cauchy principal value of an integral. Notice that this definition differs by the opposite sign from the convolution-type definition of $[9$, p. 26]. According to our choice of the Fourier transform, $\widehat{(H f})(\xi)=i \operatorname{sgn}(\xi) \widehat{f}(\xi)$. One can see that $H^{2}=-I$ on $L_{p}(\mathbf{R}), p \geq 1$, where $I$ is the identity operator.

For $x \in \mathbf{R}^{n}$ Riesz potentials are defined via the Fourier transform (see [9, p. 117] and $[10$, p. 88])

$$
\left((-\Delta)^{\alpha / 2} f\right)^{\wedge}(\xi)=|\xi|^{\alpha} \hat{f}(\xi)
$$

For $\alpha, x \in \mathbf{R}$ define the Riesz potentials by

$$
D_{x}^{\alpha}\{f(x)\}=\frac{1}{2 \pi} \int_{-\infty}^{\infty}|\xi|^{\alpha} \hat{f}(\xi) \mathrm{e}^{\mathrm{i} \xi x} \mathrm{~d} \xi
$$

provided that the integral in the right-hand side exists. Notice that for any $a>0$

$$
D_{x}^{\alpha}\{f(a x)\}=\left.a^{\alpha} D_{y}^{\alpha}\{f(y)\}\right|_{y=a x} .
$$

Introduce the function

$$
A_{2}(a, b ; x)=A i(x-a) A i(x-b) .
$$

This function appears in the studies of the Gelfand-Levitan-Marchenko equation (see [11, p. 408]), the second Painlevé equation (see [12, p. 134]) and the limit at the "edge of the spectrum" of the level spacing distribution functions obtained from scaling random models of Hermitian matrices in the Gaussian Unitary Ensemble ([13] and [14]).

\section{Asymptotic expansions of Riesz potentials of the Airy and Scorer functions for $x \rightarrow+\infty$}

The Riesz potentials of $A i(x)$ and $G i(x)$ can be written as

$$
D_{x}^{\alpha} A i(x)=\Re F(x), \quad D_{x}^{\alpha} G i(x)=\Im F(x),
$$

where $\Re f$ and $\Im f$ denote the real and imaginary parts of $f$, respectively, and

$$
F(x)=\frac{1}{\pi} \int_{0}^{\infty} \xi^{\alpha} \mathrm{e}^{\mathrm{i}\left(x \xi+\frac{1}{3} \xi^{3}\right)} \mathrm{d} \xi
$$




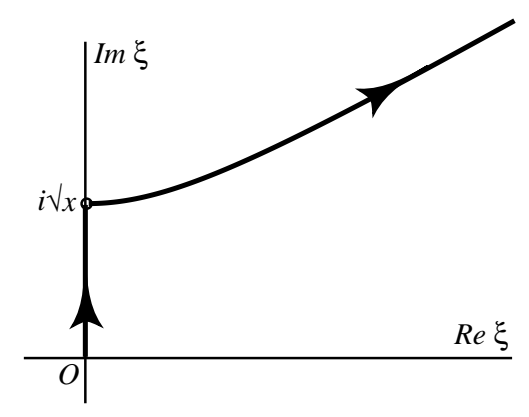

Figure 1. Modification of the path of integration giving the integral in (14) and an integral that is exponentially small.

Theorem 2 The following asymptotic expansions hold for $\alpha>-1$ and $x \rightarrow+\infty$ :

$$
D_{x}^{\alpha} A i(x) \sim \frac{\cos (\pi(\alpha+1) / 2)}{\pi x^{\alpha+1}} \sum_{k=0}^{\infty} \frac{\Gamma(\alpha+3 k+1)}{3^{k} k !} \frac{1}{x^{3 k}}
$$

where $\alpha \neq 0,2,4 \ldots$, and

$$
D_{x}^{\alpha} G i(x) \sim \frac{\sin (\pi(\alpha+1) / 2)}{\pi x^{\alpha+1}} \sum_{k=0}^{\infty} \frac{\Gamma(\alpha+3 k+1)}{3^{k} k !} \frac{1}{x^{3 k}}
$$

where $\alpha \neq 1,3,5 \ldots$

Proof We use a representation of the integral in (11) similar to the one for Gi(x) in (3.18) of [15]. To do so, notice that the exponential function in the integrand in (11) has a saddle point at $\xi=\mathrm{i} \sqrt{x}$. We integrate from the origin to this saddle point, and from there to $\infty$, inside the valley at $\infty \exp (\pi \mathrm{i} / 6)$. The latter part can be neglected, because it is exponentially small compared with the first part. Therefore we have for large positive $x$

$$
F(x)=\frac{\mathrm{e}^{\frac{1}{2} \pi \mathrm{i}(\alpha+1)}}{\pi} \int_{0}^{\sqrt{x}} v^{\alpha} \mathrm{e}^{-x v+\frac{1}{3} v^{3}} \mathrm{~d} v+\mathcal{O}\left(x^{\alpha} \mathrm{e}^{-\frac{2}{3} x^{3 / 2}}\right) .
$$

The asymptotic expansion follows from applying Watson's lemma (see [16, pp. 112-116]). We expand $\exp \left(\frac{1}{3} v^{3}\right)=\sum v^{3 k} /\left(3^{k} k !\right)$, and integrate termwise (replacing the upper limit of the interval by $\infty)$. As a result we obtain

$$
F(x) \sim \frac{\mathrm{e}^{\frac{1}{2} \pi \mathrm{i}(\alpha+1)}}{\pi} \sum_{k=0}^{\infty} \frac{1}{3^{k} k !} \int_{0}^{\infty} v^{\alpha+3 k} \mathrm{e}^{-x v} \mathrm{~d} v .
$$

Evaluating these integrals we get

$$
F(x) \sim \frac{\mathrm{e}^{\frac{1}{2} \pi \mathrm{i}(\alpha+1)}}{\pi x^{\alpha+1}} \sum_{k=0}^{\infty} \frac{\Gamma(\alpha+3 k+1)}{3^{k} k !} \frac{1}{x^{3 k}}, \quad x \rightarrow+\infty .
$$

Taking the real and imaginary parts of the last expression we deduce (12) and (13).

Remark. In order to recover the known asymptotic expansions for $\alpha=0,1,2, \ldots$ we need to complement (12) and (13) with the corresponding exponentially decaying terms from (14), that is the real and imaginary parts of the integral from $\mathrm{i} \sqrt{x}$ to $\infty \exp (\pi \mathrm{i} / 6)$. 


\section{Asymptotic expansions of Riesz potentials of the Airy and Scorer functions $\mathrm{f}$ for $\boldsymbol{x} \rightarrow-\infty$}

Theorem 3 The following asymptotic expansions hold for $x \rightarrow-\infty$ :

$$
\begin{aligned}
D_{x}^{\alpha} A i(x) \sim & \frac{|x|^{\frac{1}{2} \alpha-\frac{1}{4}} \cos \left(\frac{1}{4} \pi-\frac{2}{3}|x|^{3 / 2}\right)}{\sqrt{\pi}} \\
& -\frac{|x|^{\frac{1}{2} \alpha-\frac{1}{4}} \sin \left(\frac{1}{4} \pi-\frac{2}{3}|x|^{3 / 2}\right)\left(12 \alpha^{2}-24 \alpha+5\right)}{\sqrt{\pi} 48|x|^{3 / 2}} \\
& +\frac{\cos \left(\frac{1}{2} \pi(\alpha+1)\right)}{\pi|x|^{\alpha+1}}\left[\Gamma(\alpha+1)-\frac{\Gamma(\alpha+4)}{3|x|^{3}}+\mathcal{O}\left(\frac{1}{|x|^{6}}\right)\right]
\end{aligned}
$$

and

$$
\begin{aligned}
D_{x}^{\alpha} G i(x) \sim & \frac{|x|^{\frac{1}{2} \alpha-\frac{1}{4}} \sin \left(\frac{1}{4} \pi-\frac{2}{3}|x|^{3 / 2}\right)}{\sqrt{\pi}} \\
& -\frac{|x|^{\frac{1}{2} \alpha-\frac{1}{4}} \cos \left(\frac{1}{4} \pi-\frac{2}{3}|x|^{3 / 2}\right)\left(12 \alpha^{2}-24 \alpha+5\right)}{\sqrt{\pi} 48|x|^{3 / 2}} \\
& -\frac{\sin \left(\frac{1}{2} \pi(\alpha+1)\right)}{\pi|x|^{\alpha+1}}\left[\Gamma(\alpha+1)-\frac{\Gamma(\alpha+4)}{3|x|^{3}}+\mathcal{O}\left(\frac{1}{|x|^{6}}\right)\right] .
\end{aligned}
$$

Proof We write

$$
F(-x)=\frac{1}{\pi} \int_{0}^{\infty} \xi^{\alpha} \mathrm{e}^{\mathrm{i}\left(-x \xi+\frac{1}{3} \xi^{3}\right)} \mathrm{d} \xi
$$

and assume that in the proof $x \rightarrow+\infty$. For the integral (19) there is a positive stationary point at $\xi=\sqrt{x}$, which gives a contribution to the asymptotic expansion, but there is also a contribution from the origin. To handle both contributions, we replace the original path of integration by two new contours, giving two integrals $F(-x)=F_{1}(-x)+F_{2}(-x)$, where $F_{j}$ are defined by

$$
\begin{aligned}
& F_{1}(-x)=\frac{1}{\pi} \int_{0}^{-\mathrm{i} \infty} \xi^{\alpha} \mathrm{e}^{\mathrm{i}\left(-x \xi+\frac{1}{3} \xi^{3}\right)} \mathrm{d} \xi \\
& F_{2}(-x)=\frac{1}{\pi} \int_{-\mathrm{i} \infty}^{\infty \mathrm{e}^{\pi \mathrm{i} / 6}} \xi^{\alpha} \mathrm{e}^{\mathrm{i}\left(-x \xi+\frac{1}{3} \xi^{3}\right)} \mathrm{d} \xi
\end{aligned}
$$

So, the contour for $F_{2}$ runs from the valley at $-\mathrm{i} \infty$ to the valley at $\infty \exp (\pi \mathrm{i} / 6)$, and we can take the contour through the saddle point at $\xi=\sqrt{x}$. See Figure 2.

For $F_{1}$ we integrate by setting $\xi=-\mathrm{i} v, v>0$ and obtain

$$
F_{1}(-x)=\frac{\mathrm{e}^{-\frac{1}{2} \mathrm{i}(\alpha+1)}}{\pi} \int_{0}^{\infty} v^{\alpha} \mathrm{e}^{-\left(x v+\frac{1}{3} v^{3}\right)} \mathrm{d} v .
$$

Proceeding as for the integral in (14) we deduce that

$$
F_{1}(-x) \sim \frac{\mathrm{e}^{-\frac{1}{2} \pi \mathrm{i}(\alpha+1)}}{\pi x^{\alpha+1}} \sum_{k=0}^{\infty} \frac{\Gamma(\alpha+3 k+1)}{3^{k} k !} \frac{(-1)^{k}}{x^{3 k}}
$$

as $x \rightarrow+\infty$. 


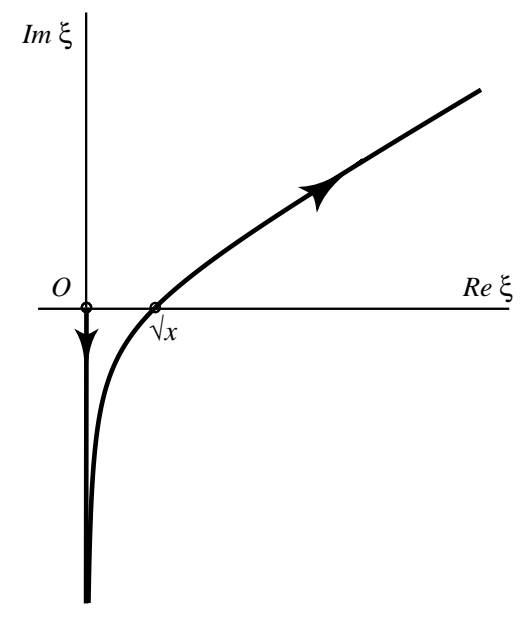

Figure 2. Modification of the path of integration giving the integrals in (20).

For $F_{2}$ we first write $\xi=\sqrt{x} \eta$, which gives

$$
\begin{aligned}
& F_{2}(-x)=\frac{x^{\frac{1}{2}(\alpha+1)}}{\pi} \int_{-\mathrm{i} \infty}^{\infty \mathrm{e}^{\pi \mathrm{i} / 6}} \xi^{\alpha} \mathrm{e}^{-x \sqrt{x} \phi(\eta)} \mathrm{d} \eta, \\
& \phi(\eta)=\mathrm{i}\left(\eta-\frac{1}{3} \eta^{3}\right) .
\end{aligned}
$$

We have $\phi(1)=\frac{2}{3} \mathrm{i}$ and $\phi^{\prime \prime}(1)=-2 \mathrm{i}$. Performing the transformation

$$
\phi(\eta)=\phi(1)+\frac{1}{2} \phi^{\prime \prime}(1) w^{2},
$$

that is

$$
\begin{aligned}
& w^{2}=\frac{2}{3}-\eta+\frac{1}{3} \eta^{3}=\frac{1}{3}(\eta+2)(\eta-1)^{2}, \\
& w=\sqrt{(\eta+2) / 3}(\eta-1),
\end{aligned}
$$

We integrate in the neighborhood of the saddle point at $w=0$ along the straight line through the origin which has an angle of $\frac{1}{4} \pi$ with the positive $w$-axis. This yields

$$
F_{2}(-x)=\frac{x^{\frac{1}{2}(\alpha+1)} \mathrm{e}^{-\frac{2}{3} x \sqrt{x} \mathrm{i}}}{\pi} \int_{\infty \mathrm{e}^{-3 \pi \mathrm{i} / 4}}^{\infty \mathrm{e}^{\pi \mathrm{i} / 4}} f(w) \mathrm{e}^{\mathrm{i} x \sqrt{x} w^{2}} \mathrm{~d} w,
$$

where

$$
f(w)=\eta^{\alpha} \frac{\mathrm{d} \eta}{\mathrm{d} w} .
$$

We expand $f(w)=\sum_{k=0}^{\infty} c_{k} w^{k}$ and deduce that

$$
F_{2}(-x) \sim \frac{x^{\frac{1}{2}(\alpha+1)} \mathrm{e}^{-\frac{2}{3} \mathrm{i} x \sqrt{x}}}{\times \sum_{k=0}^{\infty} c_{2 k} \int_{\infty \mathrm{e}^{-3 \pi \mathrm{i} / 4}}^{\infty \mathrm{e}^{\pi \mathrm{i} / 4}} w^{2 k} \mathrm{e}^{\mathrm{i} x \sqrt{x} w^{2}} \mathrm{~d} w .}
$$


To evaluate the integrals we set $w=t \mathrm{e}^{\mathrm{i} \pi / 4}$. This yields

$$
\begin{aligned}
\mathrm{e}^{\mathrm{i}\left(\frac{1}{4}+\frac{1}{2} \pi k\right)} \int_{-\infty}^{\infty} t^{2 k} \mathrm{e}^{-x \sqrt{x} t^{2}} \mathrm{~d} t & \\
= & \mathrm{e}^{\mathrm{i}\left(\frac{1}{4} \pi+\frac{1}{2} \pi k\right)} \Gamma\left(k+\frac{1}{2}\right) x^{-\frac{3}{2}\left(k+\frac{1}{2}\right)} .
\end{aligned}
$$

So, we finally obtain

$$
F_{2}(-x) \sim \frac{x^{\frac{1}{2} \alpha-\frac{1}{4}} \mathrm{e}^{\frac{1}{4} \pi \mathrm{i}-\frac{2}{3} \mathrm{i} x \sqrt{x}}}{\pi} \sum_{k=0}^{\infty} c_{2 k} \frac{\mathrm{i}^{k} \Gamma\left(k+\frac{1}{2}\right)}{x^{\frac{3}{2} k}},
$$

as $x \rightarrow+\infty$. A few first coefficients are

$$
c_{0}=1, \quad c_{2}=\frac{1}{24}\left(12 \alpha^{2}-24 \alpha+5\right) .
$$

Taking the real and imaginary parts of (21) and (28) we obtain (17) and (18).

\section{Applying the asymptotic results}

The next statement was proved in [17].

Theorem 4 The following representation holds for $x \in \mathbf{R}, a, b, \omega_{1}, \omega_{2} \in \mathbf{R}$ and $\omega_{1}, \omega_{2} \neq 0$ :

$$
\begin{array}{rl}
A i\left(\frac{x-a}{\omega_{1}}\right) A & A\left(\frac{x-b}{\omega_{2}}\right)=-\frac{2}{\Omega_{1}} \int_{0}^{\infty} J_{0}\left(2\left(\Omega_{2} x+B\right) \eta\right) \\
& \times \frac{\mathrm{d}}{\mathrm{d} x}\left[A i^{2}\left(\Omega_{1} x-A+\eta^{2}\right)\right] \eta \mathrm{d} \eta
\end{array}
$$

where

$$
\begin{array}{ll}
\Omega_{1}=\frac{\omega_{1}+\omega_{2}}{2 \omega_{1} \omega_{2}}, & \Omega_{2}=\frac{\omega_{2}-\omega_{1}}{2 \omega_{1} \omega_{2}} \\
A=\frac{a \omega_{1}+b \omega_{2}}{2 \omega_{1} \omega_{2}}, & B=\frac{b \omega_{1}-a \omega_{2}}{2 \omega_{1} \omega_{2}} .
\end{array}
$$

We list here several important corollaries that allow us to get the Hankel transforms of the function $A_{2}(a, b ; x)$ and its Riesz fractional derivatives. Notice that

$$
A i(x-a) A i(x-b)=A i(x-Y-Z) A i(x-Y+Z),
$$

where

$$
Y=\frac{a+b}{2} \quad \text { and } \quad Z=\frac{b-a}{2} .
$$

Corollary 1 The following formulas hold for $x \in \mathbf{R}$ and $a, b \in \mathbf{R}$ :

$$
A_{2}(a, b ; x)=-2 \frac{\mathrm{d}}{\mathrm{d} x} \int_{0}^{\infty} A i^{2}\left(x-Y+\eta^{2}\right) J_{0}(2 Z \eta) \eta \mathrm{d} \eta
$$

and

$$
\begin{array}{r}
-H_{x}\left\{A_{2}(a, b ; x)\right\}=-2 \frac{\mathrm{d}}{\mathrm{d} x} \int_{0}^{\infty} A i\left(x-Y+\eta^{2}\right) \\
\times B i\left(x-Y+\eta^{2}\right) J_{0}(2 Z \eta) \eta \mathrm{d} \eta .
\end{array}
$$


Proof Evidently, (33) is a particular case of (30) when $\omega_{1}=\omega_{2}=1$. Taking the Hilbert transform of (33) with respect to $x$ yields (34).

Corollary $\mathbf{2}$ For $\alpha, a, b \in \mathbf{R}$ Riesz fractional derivatives of the function $A_{2}(a, b ; x)$ are given by the formula

$$
\begin{aligned}
D_{x}^{\alpha}\left\{A_{2}(a, b ; x)\right\} & = \\
- & \frac{2^{2(\alpha-1) / 3}}{\sqrt{2 \pi}} \frac{\mathrm{d}}{\mathrm{d} x} \int_{0}^{\infty}\left[\left(D_{x}^{\alpha-1 / 2} A i\right)\left(2^{2 / 3}\left(x-Y+\eta^{2}\right)\right)\right. \\
& \left.-\left(D_{x}^{\alpha-1 / 2} G i\right)\left(2^{2 / 3}\left(x-Y+\eta^{2}\right)\right)\right] J_{0}(2 Z \eta) \eta \mathrm{d} \eta
\end{aligned}
$$

and

$$
\begin{aligned}
& H\left\{D_{x}^{\alpha}\left\{A_{2}(a, b ; x)\right\}\right\}= \\
& \quad \frac{2^{2(\alpha-1) / 3}}{\sqrt{2 \pi}} \frac{\mathrm{d}}{\mathrm{d} x} \int_{0}^{\infty}\left[\left(D_{x}^{\alpha-1 / 2} A i\right)\left(2^{2 / 3}\left(x-Y+\eta^{2}\right)\right)\right. \\
& \left.+\left(D_{x}^{\alpha-1 / 2} G i\right)\left(2^{2 / 3}\left(x-Y+\eta^{2}\right)\right)\right] J_{0}(2 Z \eta) \eta \mathrm{d} \eta
\end{aligned}
$$

where the integrals in the right-hand sides exist at least in the sense of distributions.

Proof Follows from (33) and (34).

Corollary 3 The following relations hold for $\alpha>-\frac{1}{2}$ :

$$
\begin{aligned}
2 \mathcal{H}_{Z \rightarrow \zeta}\left\{D_{x}^{\alpha-1}\right. & (A i(x-Z) A i(x+Z))\} \\
& =k_{\alpha}\left[D^{\alpha-1 / 2} A i(X)+D^{\alpha-1 / 2} G i(X)\right]
\end{aligned}
$$

and

$$
\begin{aligned}
2 \mathcal{H}_{Z \rightarrow \zeta}\left\{D_{x}^{\alpha-1} H_{x}(A i(x-Z) A i(x+Z))\right\} \\
=k_{\alpha}\left[D^{\alpha-1 / 2} A i(X)-D^{\alpha-1 / 2} G i(X)\right],
\end{aligned}
$$

where $k_{\alpha}$ is defined by (4) and $X=2^{2 / 3}\left(x+\frac{1}{4} \zeta^{2}\right)$.

Combining the asymptotic expansions (12), (13), (17) and (18) and Corollary 3 we can obtain asymptotic expansions of the Hankel transforms (37) and (38) for $x \rightarrow \pm \infty$ or $\zeta \rightarrow \infty$.

\section{Acknowledgments}

NMT acknowledges financial support from the Spanish Ministerio de Educación y Ciencia, project MTM2006-09050 and from the Gobierno of Navarra, Res. 07/05/2008. 


\section{References}

[1] Varlamov V 2005 Z. Angew. Math. Phys. 56(6) 957-985

[2] Kenig C E, Ponce G and Vega L 1989 Duke Math. J. 59(3) 585-610

[3] Kenig C E, Ponce G and Vega L 1993 Comm. Pure Appl. Math. 46(4) 527-620

[4] Hayashi N and Naumkin P I 1998 J. Funct. Anal. 159(1) 110-136

[5] Varlamov V 2008 Z. Angew. Math. Phys. 59(3) 381-399

[6] Varlamov V 2008 J. Math. Anal. Appl. 337(1) 667-685

[7] Debnath L and Bhatta D 2007 Integral Transforms and Their Applications 2nd Ed (Boca Raton, FL: Chapman \& Hall/CRC)

[8] Titchmarsh E C 1986 Introduction to the Theory of Fourier Integrals 3d Ed (New York: Chelsea Publishing Co.)

[9] Stein E M 1970 Singular Integrals and Differentiability Properties of Functions. Princeton Mathematical Series, vol 30 Princeton NJ: Princeton University Press

[10] Duoandikoetxea J 2001 Fourier Analysis, vol 29 of Graduate Studies in Mathematics. Translated and revised from the 1995 Spanish original by David Cruz-Uribe (Providence, RI: Amer. Math. Soc.)

[11] Ablowitz M J and Clarkson P A 1991 Solitons, Nonlinear Evolution Equations and Inverse Scattering, vol 149 of London Mathematical Society Lecture Note Series. (Cambridge: Camb. Univ. Press)

[12] Vallée O and Soares M 2004 Airy Functions and Applications to Physics. (Londonn: Imperial College Press)

[13] Tracy C A and Widom H 1994 Comm. Math. Phys. 159(1) 151-174

[14] Basor E L and Widom H 1999 J. Statist. Phys. 96(1-2) 1-20

[15] Gil A, Segura J and Temme N M 2001 Math. Comp. 70(235) 1183-1194 (electronic)

[16] Olver F W J 1997 Asymptotics and Special Functions. 9Wellesley, MA: AKP Classics. A K Peters Ltd.) Reprint of the 1974 original [Academic Press, New York].

[17] Varlamov V 2008 In Special Functions and Orthogonal Polynomials, vol 471 of Contemp. Math. 203-218. (Providence RI: Amer. Math. Soc.) 